\title{
Role of binding immunoglobulin protein (BiP) in Graves' orbitopathy pathogenesis
}

\author{
JaeSang Ko', Ji-Young Kim¹, Eun Jig Lee² and Jin Sook Yoon ${ }^{1}$ \\ 1Department of Ophthalmology, Severance Hospital, Institute of Vision Research, Yonsei University College of Medicine, Seoul, Korea \\ 2Department of Endocrinology, Severance Hospital, Yonsei University of Medicine, Seoul, Korea
}

Correspondence should be addressed to J S Yoon: yoonjs@yuhs.ac

\begin{abstract}
Inflammation and adipogenesis represent the main pathogenic mechanisms of Graves' orbitopathy (GO), and oxidative stress is a well-known inducer of GO pathology. Endoplasmic reticulum (ER) stress has been suggested as a major contributor to inflammation and reactive oxygen species (ROS) generation. In this study, we investigated the role of the ER-stress chaperone protein, binding immunoglobulin protein (BiP), in $\mathrm{GO}$ pathogenesis. Using primary cultures of orbital fibroblasts from patients with $\mathrm{GO}$, we examined the role of $\mathrm{BiP}$ in $\mathrm{GO}$ pathogenesis by silencing its expression with smallinterfering RNA (siRNA). Inflammatory cytokine expression was analysed by Western blotting and ELISA. Intracellular ROS levels induced by hydrogen peroxide or cigarette smoke extract were measured by 5-(and 6)-carboxy-20,70-dichlorodihydrofluorescein diacetate staining and flow cytometry. After adipogenic differentiation in BiP siRNAtransfected cells, the cells were stained with Oil Red O, and the levels of adipogenic transcription factors were determined by Western blot analysis. BiP mRNA expression levels were significantly higher in GO orbital tissues than in non-GO orbital tissues. Silencing BiP attenuated the expression of pro-inflammatory cytokines (interleukin-6, intercellular adhesion molecule-1, and monocyte chemotactic protein-1) in primary cultured GO orbital fibroblasts. Silencing BiP also reduced ROS generation, hyaluronan production, and adipocyte differentiation. These findings suggest that ER stress is involved in the aetiology of GO and that modulation of ER stress has therapeutic potential for GO.
\end{abstract}

\author{
Key Words \\ - binding immunoglobulin \\ protein \\ - endoplasmic reticulum \\ stress \\ - Graves' orbitopathy \\ - thyroid eye disease \\ - unfolded protein \\ response
}

\section{Introduction}

Graves' orbitopathy (GO) is an autoimmune disorder of the orbits involving infiltration by T cells, B cells, plasma cells, and macrophages; these features are pathologic manifestations of Graves' disease, an autoimmune disorder of the thyroid gland (Garrity \& Bahn 2006, Bahn 2010). Also known as thyroid eye disease, GO presentation includes ocular pain, eyelid retraction, oedema of periorbital tissues, proptosis, and compressive optic neuropathy. Orbital fibroblasts have been found to be the key for the progression of this disease, and cellular immunity plays an important role in orbital inflammation during GO pathogenesis (Khong et al. 2016). Multiple intracellular signalling cascades lead to hyaluronic acid secretion, adipogenesis, and the release of chemotactic factors and cytokines, ensuring the perpetuation of orbital inflammation (Bahn 2010, Khong et al. 2016). 
The endoplasmic reticulum (ER) is responsible for the folding of secreted and membrane-bound proteins (Kaufman 1999). In the ER, unfolded or misfolded proteins are detected and retained until they are properly folded or degraded. Under conditions that challenge ER function (particularly an increase in newly synthesised, unfolded proteins in the ER lumen), unfolded proteins accumulate in the ER, reflecting cellular stress induced by multiple stimuli and pathological conditions (Kim et al. 2008). Under this condition, the ER elicits an elaborate adaptive response known as the unfolded protein response (UPR), which intersects with various inflammatory and stress signalling pathways (Ron \& Walter 2007, Hotamisligil 2010, Dandekar et al. 2015).

Accumulating evidence suggests that ER stress plays roles in autoimmune and inflammatory diseases, such as rheumatoid arthritis, systemic lupus erythematosus, and idiopathic inflammatory myopathies (Todd et al. 2008, Park et al. 2014, Lee et al. 2015, Lightfoot et al. 2015). Moreover, ER stress activation promotes inflammation-mediated proteolytic activity in ocular-surface diseases, such as Sjögren's syndrome and ocular cicatricial pemphigoid (Barrera et al. 2018, Woodward et al. 2020). Although ER stress is known to be involved in inflammation-associated diseases, its involvement in the inflammatory process of GO has not been investigated. Therefore, we studied the role of ER stress in GO pathogenesis by modulating binding immunoglobulin protein (BiP, an ER stress chaperone protein known as the ER stress sensor; also known as GRP78 or HSPA5) in primary cultured orbital fibroblasts and evaluated the potential of ER stress as a therapeutic target.

\section{Materials and methods}

\section{Reagents and chemicals}

Dulbecco's modified Eagle's medium (DMEM), foetal bovine serum (FBS), penicillin, and gentamicin were purchased from Hyclone Laboratories (Logan, UT, USA). BiP short interfering RNA (siRNA; \#4392420) and negative control siRNA (\#4390843) were purchased from Ambion/ Applied Biosystems. An anti-BiP antibody was purchased from Abcam. Antibodies against interleukin (IL)-6, IL-8, peroxisome proliferator-activator gamma (PPAR $\gamma$ ), CCAAT-enhancer-binding protein (C/EBP) $\alpha, \mathrm{C} / \mathrm{EBP} \beta$, and $\beta$-actin were obtained from Santa Cruz Biotechnology. Antibodies against nuclear factor kappa-light-chainenhancer of activated B cells (NF-kB), c-Jun NH(2)terminal kinase (JNK), intercellular adhesion molecule (ICAM)-1, and monocyte chemotactic protein (MCP)-1 were produced by Cell Signaling Technology. ELISA kits for IL-6, IL-8, MCP-1, and hyaluronan were purchased from R\&D Systems, and an ICAM-1 ELISA kit was purchased from Abcam. Recombinant human IL-1 $\beta$ was purchased from R\&D Systems, and Oil Red O was purchased from Sigma-Aldrich. Cigarette smoke extract (CSE) was freshly prepared within $1 \mathrm{~h}$ before each experiment from commercially available filtered cigarettes (Marlboro 20 class A cigarettes (8.0 mg tar; $0.7 \mathrm{mg}$ nicotine); Philip Morris Korea, Inc.), as described in our previous study (Yoon et al. 2013).

\section{Cell culture and differentiation protocols}

Orbital adipose/connective tissue explants were obtained as surgical waste during decompression surgery from ten patients with GO (seven females, three males; 25-65 years of age). Normal control tissues were harvested during upper-lid blepharoplasty from the post-septal area of seven individuals with no history or clinical evidence of thyroid disease or GO (five females, two males; 31-61 years of age). All ten patients with GO had stable euthyroidism at the time of surgery, when their clinical-activity scores were less than three. Furthermore, no patients with GO were administered steroids, other types of immunomodulatory treatments, or radiotherapy for at least three months before surgery. The Institutional Review Board of Severance Hospital, Yonsei University College of Medicine (Seoul, Korea) approved the study (approval number 4-2019-1025), and written informed consent was obtained from all participants after explanation of the nature and possible consequences of the study. This study followed the tenets of the Declaration of Helsinki.

Primary cultures of orbital fibroblasts were established as described in our previous study (Kim et al. 2016). Briefly, minced tissue was placed directly in 1:1 DMEM:F12 medium containing 20\% FBS and antibiotics. When observing fibroblast growth, monolayers were passaged serially with trypsin/ethylenediaminetetraacetic acid solution, and the cell cultures were maintained in DMEM containing $10 \% \mathrm{FBS}$ and antibiotics. The strains were stored in liquid nitrogen until further analysis; cells between the second and fifth passages were used. The adipogenic potential of orbital fibroblasts was evaluated using our previously reported adipocyte-differentiation protocol for GO orbital fibroblasts involving dexamethasone, rosiglitazone (10 $\mu \mathrm{mol} / \mathrm{L}$; Cayman Chemical), and IL-1 $\beta$ (10 ng/mL) treatment (Kim et al. 2016, Ko et al. 2018, 2020). 


\section{Real-time polymerase chain reaction (PCR) analysis}

BiP expression levels in orbital tissues from healthy controls and individuals with GO were evaluated by real-time PCR. RNA isolation from orbital tissues and real-time PCR were performed as described previously (Kim et al. 2016). RNA was extracted from seven GO and control orbital tissues using TriZol reagent (Invitrogen), $1 \mathrm{~g}$ of which was reversetranscribed into cDNA (Qiagen). The cDNA was then amplified with TaqMan universal PCR master mix in an ABI 7300 real-time PCR thermocycler (Applied Biosystems). All PCR experiments were performed in triplicate, and the BiP mRNA expression levels in all samples were normalised to glyceraldehyde-3-phosphate dehydrogenase (GAPDH)expression levels. Expression levels were determined by the $2^{-\Delta \Delta \mathrm{Ct}}$ method, as the fold-change of the threshold cycle (Ct) value between healthy subjects and patients with GO, relative to the expression of the GAPDH control. The amplification primers were as follows: human BiP 5'-TGTTCAACCAATTATCAGCAAACTC-3' (forward) and 5'-TTCTGCTGTATCCTCTTCACCAGT-3' (reverse), and GAPDH 5'-GCCAAGGTCATCCATGACAAC-3' (forward) and 5'-GTCCACCACCCTGTTGCTGTA-3' (reverse).

\section{siRNA transfections}

Orbital fibroblasts from patients with GO were cultured to $\sim 80 \%$ confluency in $100-\mathrm{mm}$ plates and transfected with BiP siRNA or negative-control siRNA using the TransIT-siQUEST reagent (Mirus, PanVera, Madison, WI, USA) according to the manufacturer's instructions. After transfection, inflammation was simulated by treatment with IL-1 $\beta(10 \mathrm{ng} / \mathrm{mL})$ or oxidative stress was induced either with CSE $(2 \%)$ or $\mathrm{H}_{2} \mathrm{O}_{2}(200 \mu \mathrm{mol} / \mathrm{L})$ for further investigation. To evaluate the adipogenic differentiation potential of the transfectants, siRNA-transfected cells were differentiated for 10 days as described previously.

\section{Western blot analysis}

Western blot analysis was performed as described previously (Byun et al. 2016, Lee et al. 2019). To assess the attenuation of pathogenic GO mechanisms under BiP silencing, confluent orbital fibroblasts were transfected with BiP siRNA or negative-control siRNA and cultured for $24 \mathrm{~h}$. To evaluate the effect of BiP silencing on the intracellular expression of inflammatory mediators, we evaluated IL-6, IL-8, ICAM-1, and MCP-1 expression after exposing the transfected cells to $10 \mathrm{ng} / \mathrm{mL}$ IL- $1 \beta$ to induce inflammation for 24 or $48 \mathrm{~h}$. To evaluate the expression of adipogenic transcription factors after 10 days of adipogenic differentiation, we evaluated PPAR $\gamma, \mathrm{C} / \mathrm{EBP} \alpha$, and C/EBP $\beta$ expression in transfected GO cells. The activation of intracellular signalling proteins was assayed by Western blot analysis of NF- $\mathrm{kB}$ and JNK protein expression. The relative levels of protein in each immunoreactive band were quantified by densitometry and normalisation to the $\beta$-actin concentration in the same sample.

\section{ELISA}

Fibroblast supernatants were collected for ELISAs against IL-6, IL-8, ICAM-1, MCP-1, and hyaluronan, which were performed as described previously (Yoon et al. 2012, Ko et al. 2018). After measuring the absorbance at $405 \mathrm{~nm}$ and determining the percentage of binding for each sample, a standard binding curve was generated to determine the cytokine concentrations. Samples were diluted 1:10 before analysis, and the average value of three assays was used for statistical analyses.

\section{Intracellular reactive oxygen species (ROS) measurements}

ROS release was determined using 5-(and 6)-carboxy20,70-dichlorodihydrofluorescein diacetate (H2DCFDA; Invitrogen), an oxidant-sensitive fluorescent probe as described in our previous study (Yoon et al. 2013). Briefly, primary cultured orbital fibroblasts were transfected with BiP siRNA or negative-control siRNA and cultured for $24 \mathrm{~h}$. The culture medium was then removed, and the cells were washed with PBS, incubated with $10 \mathrm{mM} \mathrm{H2DCFDA} \mathrm{at}$ $37^{\circ} \mathrm{C}$ for $30 \mathrm{~min}$, and stimulated with CSE $(2 \%)$ or $\mathrm{H}_{2} \mathrm{O}_{2}$ ( $200 \mu \mathrm{mol} / \mathrm{L}$ ) for $30 \mathrm{~min}$ (Yoon et al. 2013, Kim et al. 2015, Ko et al. 2018, 2020). Fluorescently stained cells were examined microscopically at $40 \times$ and quantified using flow cytometric analysis.

\section{Oil Red O staining}

After 10 days of differentiation of siRNA-transfected orbital fibroblasts, the cells were stained with Oil Red $\mathrm{O}$ as described previously (Kim et al. 2016, Ko et al. 2018). Stained cells were visualised and photographed at 40× magnification and then cell-bound Oil Red O was solubilised with $100 \%$ isopropanol; the optical density of the solution was measured using a spectrophotometer at $490 \mathrm{~nm}$. Quantitative assessments of adipogenic differentiation were performed in duplicate using cells from different donors; the results were normalised to the 
absorbance of differentiated control cells transfected with negative-control siRNA.

\section{Statistical analysis}

All experiments were performed using cells from at least three different samples, which were assayed in duplicate. All outliers have been included. Differences in parameter estimates between the experimental and control groups were assessed by Student's t-test or Wilcoxon's rank-sum test using $\mathrm{R}$ version 3.1.2 (R Foundation, Vienna, Austria). Values of $P<0.05$ were considered significant.

\section{Results}

\section{BiP mRNA expression in orbital tissues}

To evaluate BiP expression in whole tissue specimens from the orbital adipose connective tissues of patients with GO and control subjects, we quantified the mRNA expression levels of BiP by real-time PCR. Orbital tissues acquired from seven patients with GO and seven healthy control subjects were used. The BiP mRNA expression levels were significantly higher in GO orbital tissues than in non-GO orbital tissues $(P<0.001$, Fig. 1$)$.

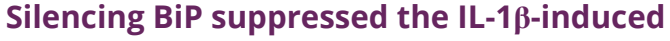 expression of pro-inflammatory cytokines}

To evaluate the anti-inflammatory potential of silencing BiP, orbital fibroblasts from patients with GO were transfected with BiP-targeted siRNA followed by IL-1 $\beta$ treatment $(10 \mathrm{ng} / \mathrm{mL})$ for $24 \mathrm{~h}$ or $48 \mathrm{~h}$. Western blot

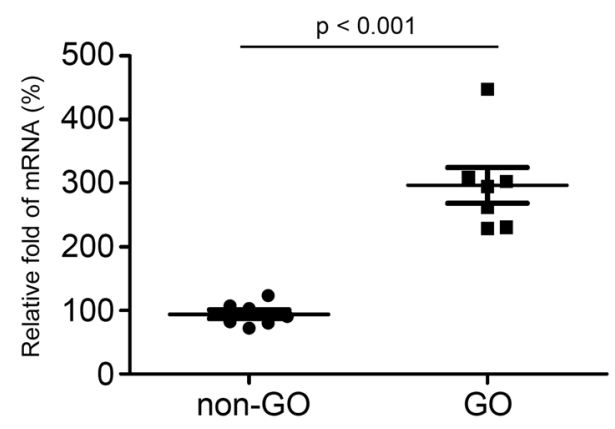

\section{Figure 1}

BiP mRNA expression in GO and non-GO orbital tissues. Orbital tissues were obtained from GO patients $(n=7)$ and normal subjects without GO $(n=7)$. BiP mRNA expression levels were measured by real-time PCR and compared between patients with GO and healthy controls. Orbital tissue from patients with GO showed significantly higher BiP mRNA levels than that from non-GO controls $(P<0.001)$. analysis revealed that transfection with BiP siRNA down-regulated $\mathrm{BiP}$ expression. IL-1 $\beta$-induced IL-6 and ICAM-1 expression decreased significantly in BiPknockdown cells (Fig. 2). ELISA confirmed the suppressive
A
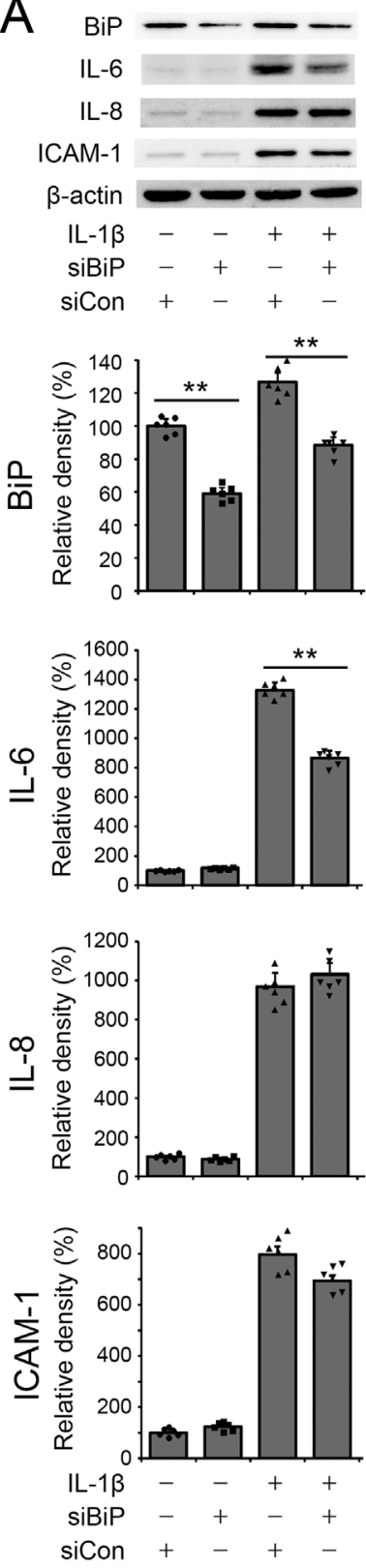
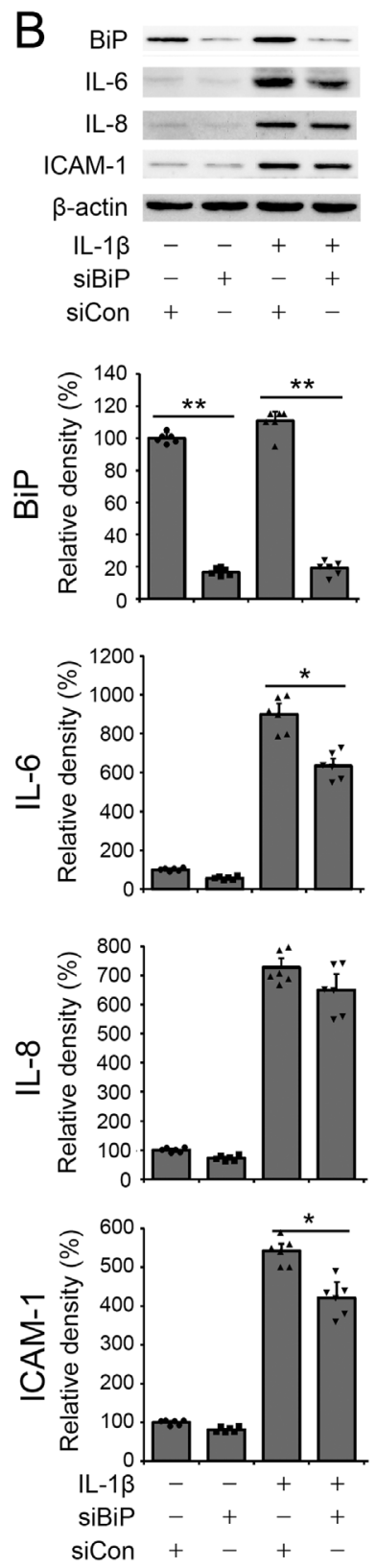

Figure 2

Suppressive effect of BiP silencing on the expression of pro-inflammatory proteins in GO orbital fibroblasts. Orbital fibroblasts from patients with $\mathrm{GO}(n=3)$ were transfected with BiP siRNA (siBiP) or negative-control SiRNA (siCon) and cultured for $24 \mathrm{~h}$, followed by treatment with IL-1 $\beta$ (10 ng/mL) for $24 \mathrm{~h}(\mathrm{~A})$ or $48 \mathrm{~h}$ (B). BiP, IL-6, IL-8, and ICAM-1 levels in transfected cells were analysed by Western blotting. The data shown in the columns indicate the mean relative density ratio \pm S.D. of three independent experiments ( $* P<0.05$ and $* * P<0.01$ vs negative-control siRNA transfectants) 
effect of BiP silencing on pro-inflammatory cytokine production (IL-6, ICAM-1, and MCP-1) in GO orbital fibroblasts (Fig. 3).

\section{Silencing BiP suppressed ROS levels induced by $\mathrm{H}_{2} \mathrm{O}_{2}$ or CSE}

To demonstrate the anti-oxidative effect of silencing BiP mRNA in GO orbital fibroblasts, oxidative stress was induced by $200 \mu \mathrm{mol} / \mathrm{L} \mathrm{H}_{2} \mathrm{O}_{2}$ or $2 \%$ CSE in orbital fibroblasts transfected with BiP or negative-control siRNA. Silencing BiP attenuated the ROS production induced by $200 \mu \mathrm{mol} / \mathrm{L} \mathrm{H}_{2} \mathrm{O}_{2}$ or $2 \%$ CSE (Fig. 4).

\section{Silencing BiP reduced adipogenesis in $\mathrm{GO}$ orbital fibroblasts}

To determine the effect of BiP silencing on adipogenesis in orbital fibroblasts, orbital fibroblasts from patients with GO were transfected with BiP-targeted siRNA followed by 10 days of adipocyte differentiation with or without IL-1 $\beta$ treatment. Silencing BiP reduced the number of adipocytes and suppressed lipid droplet accumulation. The optical density of Oil Red O-stained cell lysates showed that BiP siRNA transfectants exhibited decreased absorbance at 490 $\mathrm{nm}$ (Fig. 5A). Western blot analysis showed that silencing BiP inhibited the IL-1 $\beta$-induced expression of adipogenic transcription factors (PPAR $\gamma, \mathrm{C} / \mathrm{EBP} \alpha$, and $\mathrm{C} / \mathrm{EBP} \beta)$ during adipogenesis (Fig. 5B). Each experiment was performed in GO cell samples from three different patients, and the samples were assayed in duplicate.

\section{Silencing BiP reduced hyaluronan production in $\mathrm{GO}$ orbital fibroblasts}

To evaluate the effect of silencing $\mathrm{BiP}$ on hyaluronan production, orbital fibroblasts from patients with or without GO were transfected with BiP-targeted siRNA followed by IL-1 $\beta$ treatment $(10 \mathrm{ng} / \mathrm{mL})$ for 24 or $48 \mathrm{~h}$. ELISA using the supernatant revealed that BiP silencing suppressed hyaluronan production in GO and non-GO orbital fibroblasts (Fig. 6). Moreover, more robust hyaluronan production was induced by IL- $1 \beta$ in GO orbital fibroblasts than in non-GO cells.

\section{Intracellular signalling pathways related to ER-stress responses}

To investigate the signalling pathways through which BiP mediates its effects in GO orbital fibroblasts, the expression levels of multiple transcription factors were assessed after BiP siRNA transfection. Silencing BiP attenuated the IL-1 $\beta$ induced phosphorylation of NF-kB, whereas it did not affect JNK expression (Fig. 7).
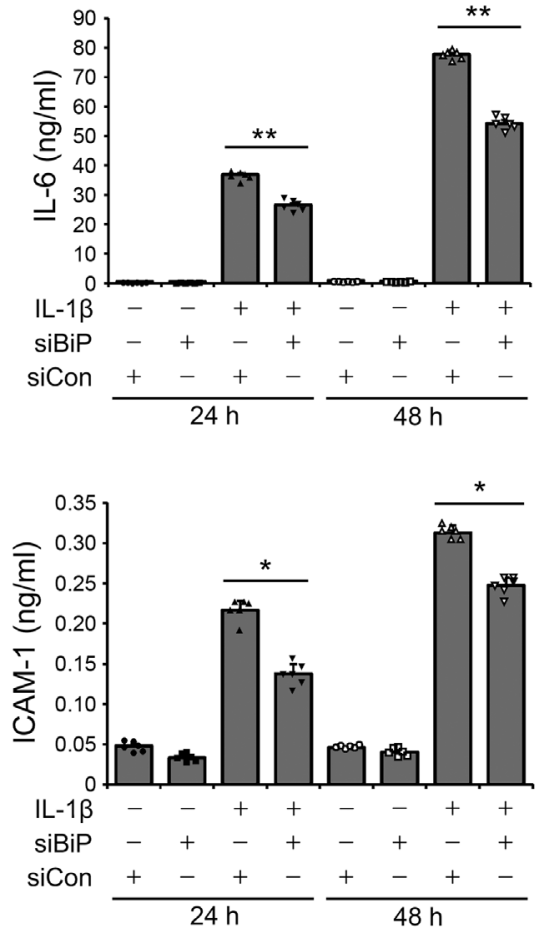
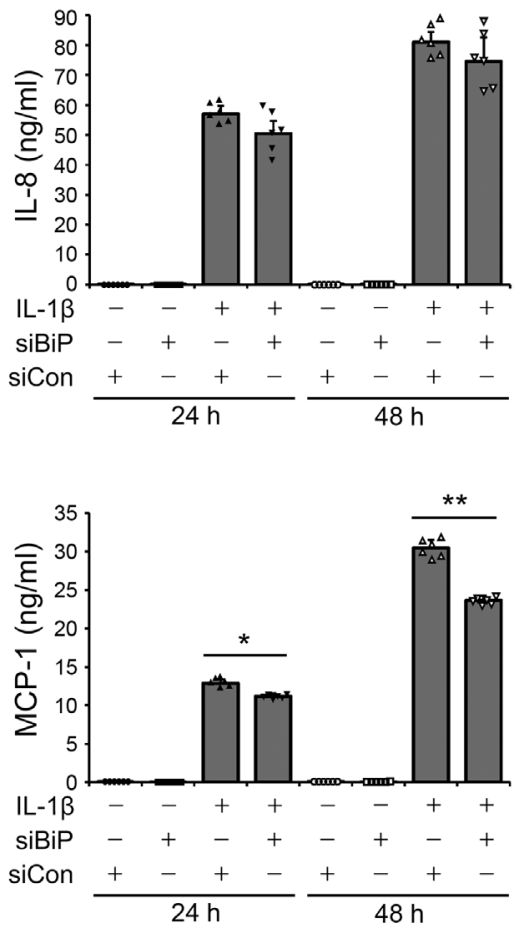

Figure 3

Effect of BiP silencing on the excretion of pro-inflammatory cytokines in GO orbital fibroblasts. Orbital fibroblasts from patients with $\mathrm{GO}(n=3)$ were transfected with BiP siRNA (siBiP) or negative-control siRNA (siCon) and cultured for $24 \mathrm{~h}$, followed by treatment with IL-1 $\beta(10 \mathrm{ng} / \mathrm{mL})$ for 24 or 48 h. IL-6, IL-8, ICAM-1, and MCP-1 were analysed in supernatants from BiP siRNA transfectants treated with IL-1 $\beta(10 \mathrm{ng} / \mathrm{mL})$, using ELISA. The data shown in the columns indicate the mean \pm S.D. of three independent experiments ( $* P<0.05$ and $* * P<0.01$ vs negative-control SiRNA transfectants). 

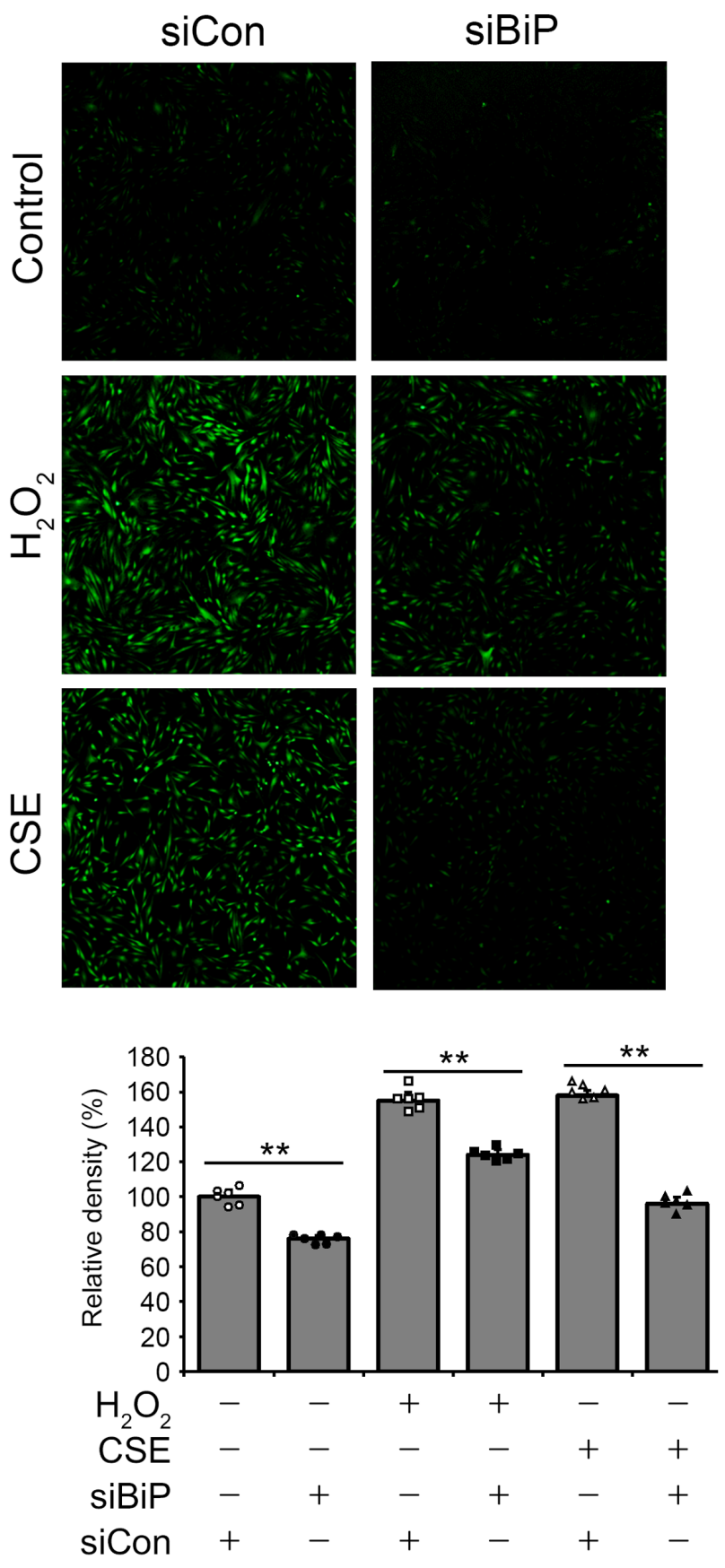

\section{Figure 4}

Effect of BiP silencing on the $\mathrm{H}_{2} \mathrm{O}_{2}$ - and CSE-induced generation of intracellular ROS in orbital fibroblasts from patients with GO. Orbital fibroblasts from patients with $\mathrm{GO}(n=3)$ were transfected with BiP siRNA ( $\mathrm{SiBiP}$ ) or negative-control siRNA (siCon) and cultured for $24 \mathrm{~h}$, followed by treatment with $200 \mu \mathrm{mol} / \mathrm{L} \mathrm{H}_{2} \mathrm{O}_{2}$ or $2 \%$ CSE for $30 \mathrm{~min}$. ROS levels were measured by flow cytometry after H2DCFDA staining and visualised microscopically at 40× magnification. The results are expressed as percentages of the control values and are presented as the mean relative density ratio \pm S.D. of three independent experiments. Assays were performed in duplicate with cells from three different GO samples; data from a representative experiment are shown ( $* * P<0.01$ vs negativecontrol siRNA transfectants). A full colour version of this figure is available at https://doi.org/10.1530/JME-20-0155.

\section{Discussion}

Here, we found that the level of a representative ER chaperone protein, $\mathrm{BiP}$, was up-regulated in orbital tissues from patients with GO compared to in tissues from healthy control subjects, indicating that ER stress was higher in the orbital tissue of patients with GO. BiP knockdown via siRNA transfection curtailed inflammation, ROS generation, and adipogenesis, which are the main pathogenic mechanisms underlying GO.

In eukaryotic cells, monitoring of the ER lumen and signalling through the canonical branches of the UPR are mediated by three ER membrane-associated proteins, namely PKR-like eukaryotic initiation factor 2a kinase (PERK), inositol-requiring enzyme 1 (IRE1), and activating transcription factor-6 (ATF). BiP is a central component of the UPR (Zha \& Zhou 2012). In a well-functioning and 'stress-free' ER, the three aforementioned transmembrane proteins are bound by $\mathrm{BiP}$ in their intraluminal domains and rendered inactive (Bertolotti et al. 2000, Hotamisligil 2010). Under ER stress, BiP interacts with unfolded proteins, promoting the reversible dissociation of BiP from the lumenal domains of these transmembrane proteins, thereby leading to the activation of these UPR transducers (Bertolotti et al. 2000, Flamment et al. 2012). The three branches of the canonical UPR intersect with various inflammatory and stress-signalling systems, including the NF- $\mathrm{KB}$ and JNK pathways, as well as networks activated by oxidative stress. In this study, we found that UPR blockage by BiP depletion attenuated the NF- $\mathrm{B}$ pathway and spared the JNK pathway in an in vitro model of GO.

Accumulating evidence has suggested that immune responses are adversely affected by abnormalities in the UPR, which may contribute to the development of autoimmunity (Todd et al. 2008). Various models suggest that an inadequate UPR and aberrant protein folding contribute to autoimmunity through multiple mechanisms, such as the recognition of misfolded proteins by autoreactive immune cells and release of neo-autoantigens and UPR-related autoantigens (such as $\mathrm{BiP}$ ). As a representative ER chaperone, BiP acts as an ER-stress sensor and can elicit autoreactive B- and T-cell responses in humans and rodents with autoimmune disease (Todd et al. 2008). Autoantibodies against BiP were present in $80 \%$ of patients with rheumatoid arthritis, and they preferentially stimulated increased proliferation of synovial T cells (Blass et al. 2001, Corrigall et al. 2001). ER stress in the instantaneous down-regulation of MHC class I molecules on thyrocytes and thyrocytes undergoing ER stress stimulates pro-inflammatory cytokines, suggesting 
A
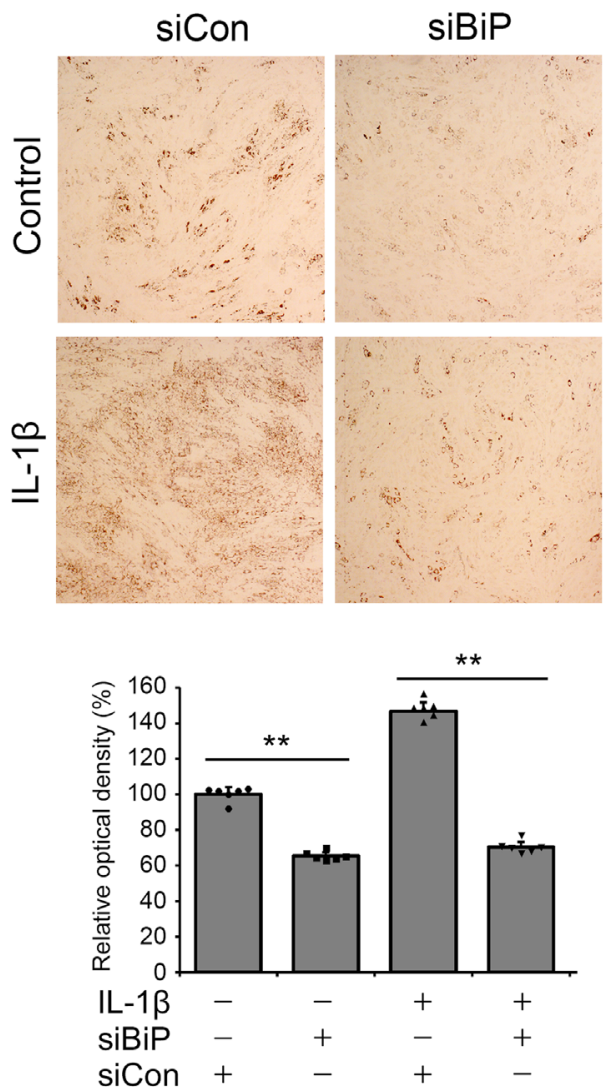

B
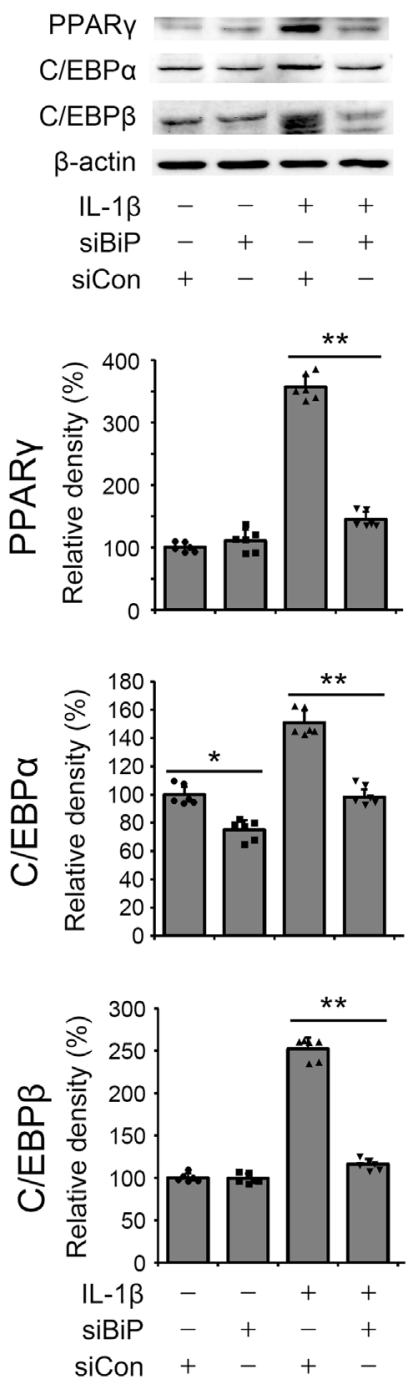

\section{Figure 5}

Effect of BiP silencing on adipogenesis in GO orbital fibroblasts. (A) Adipocyte differentiation was induced for 10 days in BiP siRNA (siBiP) or negative-control siRNA (siCon)-transfected orbital fibroblasts from patients with GO. Adipocyte differentiation was evaluated by microscopy (40x) after Oil Red $O$ staining. The optical density at 490 $\mathrm{nm}$ of stained cell lysates was also measured for quantification. (B) Western blot analysis of PPAR $\gamma$, $C / E B P \alpha$, and $C / E B P \beta$ protein levels was performed after 10 days of adipogenic differentiation. The experiments were performed in duplicate with cells from three different donors. PPAR $\gamma, C / E B P \alpha$, and C/EBP $\beta$ levels were determined by densitometry and normalised to the $\beta$-actin expression levels in the same samples. The results are presented as the mean relative density ratio (\%) \pm S.D. of three independent experiments $(* * P<0.01$ vs negative-control siRNA

transfectants). A full colour version of this figure is available at https://doi.org/10.1530/JME-20-0155. a role for ER stress in the pathogenesis of autoimmune thyroid diseases (Ulianich et al. 2011). Moreover, proteomic analysis revealed aberrant expression of BiP in thyroid tissues of patients with Graves' disease (Meng et al. 2017). BiP expression in the orbital tissue of patients with GO was increased compared that in the orbital tissue from non-GO control subjects, and this may be related to GO pathogenesis (Fig. 1). Therefore, further analysis was performed to evaluate the effect of silencing overexpressed $\mathrm{BiP}$ in GO orbital fibroblasts on GO pathogenesis. As BiP expression in non-GO orbital tissues remained low, analysing the effect of BiP silencing in non-GO orbital fibroblasts was considered unnecessary. In this study, IL-1 $\beta$-induced expression of pro-inflammatory cytokines was attenuated by silencing BiP in an in vitro model of $\mathrm{GO}$, indicating that BiP participated in inflammatory GO pathogenesis. Although UPR blockage by BiP depletion attenuated the NF-кB pathway and decreased the expression of IL-6, ICAM-1, and MCP-1, unexpectedly, the expression of IL- 8 showed no statistically significant change. IL- 8 is involved in the inflammatory pathogenesis of GO by inducing chemotaxis in neutrophils and leukocytes to the orbital tissue, and it is mainly controlled by the NF- $\mathrm{B}$ pathway (Douglas et al. 2014). In Figs 2B and 3, a decrease in IL-8 levels following siBiP transfection was observed to some extent in IL-1 $\beta$-treated cells; however, the difference was not statistically significant. This may be because the degree of NF-кB down-regulation by BiP silencing was not robust (Fig. 7), and intracellular signalling other than $\mathrm{NF}-\kappa \mathrm{B}$ and BiP may be involved in IL-8 expression in GO orbital fibroblasts.

The ER lumen is a highly oxidising environment that promotes disulphide bond formation. It has been estimated that $25 \%$ of the ROS generated in a cell may result from disulphide bond formation in the ER during oxidative protein folding ( $\mathrm{Tu} \&$ Weissman 2004). 

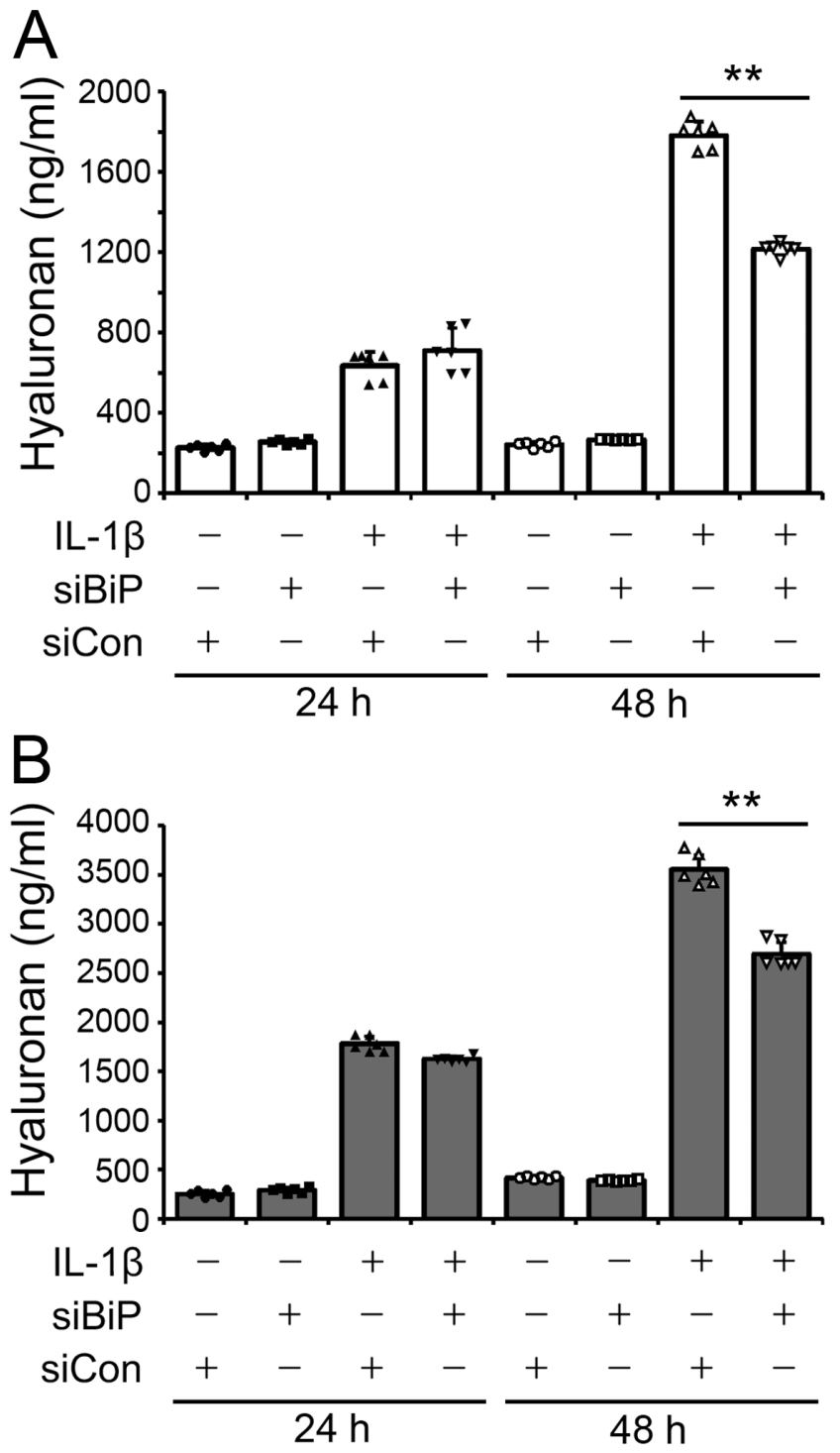

Figure 6

Effect of BiP silencing on the production of hyaluronan in GO and non-GO orbital fibroblasts. Confluent orbital fibroblasts derived from individuals with (grey columns, $n=3$ ) and without (white columns, $n=3$ ) GO were transfected with BiP siRNA (siBiP) or negative-control siRNA (siCon) and cultured for $24 \mathrm{~h}$, followed by treatment with IL-1 $\beta(10 \mathrm{ng} / \mathrm{mL})$ for 24 or $48 \mathrm{~h}$. Hyaluronan levels were analysed in the supernatants from BiP siRNA transfectants treated with IL-1 $\beta(10 \mathrm{ng} / \mathrm{mL})$ using ELISA. The data shown in the columns indicate the mean \pm S.D. of three independent experiments ( $* * P<0.01$ vs negative-control siRNA transfectants).

Accumulating evidence suggests that protein folding and ROS generation, as by-products of protein oxidation in the ER, are closely linked events. It has also become apparent that UPR activation upon exposure to oxidative stress is an adaptive mechanism for preserving cell functions and survival (Malhotra \& Kaufman 2007). Moreover, oxidative stress can disrupt the protein-folding mechanism and enhance the production of misfolded
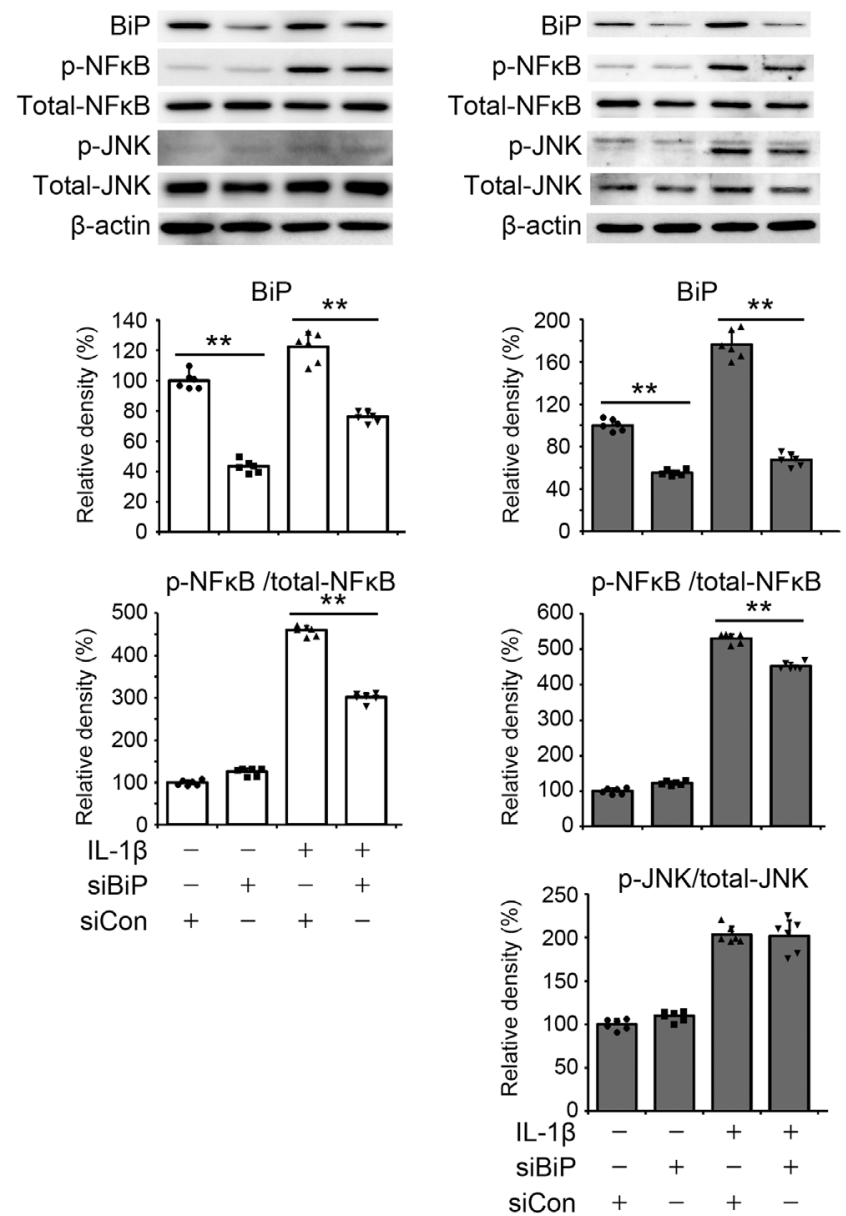

Figure 7

Effect of BiP silencing on intracellular signalling pathways. Confluent orbital fibroblasts derived from individuals with (grey columns, $n=3$ ) and without (white columns, $n=3$ ) GO were transfected with BiP siRNA (siBiP) or negative-control siRNA (siCon) and cultured for $24 \mathrm{~h}$, followed by treatment with IL-1 $\beta(10 \mathrm{ng} / \mathrm{mL})$ for $1 \mathrm{~h}$. Total and phosphorylated NF-kB and JNK were assayed by Western blot analysis. The data shown in the columns indicate the mean relative density ratio \pm S.D. of three independent experiments ( $* * P<0.01$ vs negative-control siRNA transfectants).

proteins, causing further ER stress (Chong et al. 2017). As the state of oxidative stress has been described in GO, and inflammation and oxidative stress are central to GO pathogenesis (Ko et al. 2020), the anti-oxidative effects of BiP silencing in our study indicate that modulating ER stress with BiP can block the vicious cycle of oxidative stress in GO.

The potential roles of the ER in regulating lipogenesis and the lipid droplet number, composition, and size have been suggested. In multiple studies, lipid droplets isolated from cells have been shown to contain BiP, supporting its ER origin (Prattes et al. 2000). Moreover, chronic inflammation of adipose tissue (and recruitment 
of immune cells to this site) has been linked to a diverse set of stress responses emerging from the ER (Hotamisligil 2010). Particularly, BiP appears to be essential for adipocyte differentiation in vitro, as evidenced by the fact that BiP deletion results in a 90\% reduction in fat atrophy in adipose tissue (Zhu et al. 2013). In this study, we showed that silencing BiP attenuated adipogenic differentiation and lipid accumulation, as well as the expression of adipogenic transcription factors (PPAR $\gamma, \mathrm{C} / \mathrm{EBP} \alpha$, and $\mathrm{C} / \mathrm{EBP} \beta)$.

The downstream consequences of transduction through three UPR-signalling axes require further analysis. It has been suggested that IRE1-mediated UPR signalling enables cells to adapt to stress by initiating autophagy, a survival mechanism that eliminates abnormal proteins and damaged organelles (Maiuri et al. 2007). In addition, JNK activation can promote apoptosis in cells with irrecoverable levels of ER stress by activating caspase signalling (Yoneda et al. 2001). Activation of PERKmediated UPR signalling arrests most mRNA translation to alleviate ER stress from additional sources (Harding et al. 2000). ATF6 activation during ER stress is related to autophagy induction, vesicle nucleation, and elongation of the phagophore, which induces proteolytic activation (Todd et al. 2008). Together, the three UPR-signalling axes form a coordinated response to stress caused by unfolded proteins (Todd et al. 2008). Although the findings of this study suggest that ER stress is involved in the aetiology of $\mathrm{GO}$, further studies are needed to evaluate the downstream pathways of ER stress signalling in orbital fibroblasts from patients with GO.

Orbital tissues in patients with GO are continuously exposed to oxidative stress and pro-inflammatory stimuli from infiltrated immune cells, predominantly T-lymphocytes (Garrity \& Bahn 2006, Hondur et al. 2008). Cultured orbital fibroblasts derived from individuals with an inactive status may not directly reflect the in vivo inflammatory or oxidative conditions (Wakelkamp et al. 2003, Ko et al. 2017). However, rehabilitative surgical therapy, such as decompression, is typically best initiated after six months of stable, inactive thyroid eye disease (Bartalena et al. 2008). If decompression surgery is performed during the active phase, further proptosis may occur because of inflammatory changes that persist post-operatively (Bothun et al. 2009). Because of this limitation, oxidative or pro-inflammatory conditions are inevitably induced by stimulants in cultured orbital fibroblasts to induce pathogenic changes (Ko et al. 2017, 2018). In the present study, we simulated oxidative or pro-inflammatory conditions in in vivo orbital tissues by stimulating cultured cells with $\mathrm{H}_{2} \mathrm{O}_{2}$, CSE, or IL- $1 \beta$. Silencing BiP reduced the production of intracellular ROS in non-stimulated cells. However, in most cases, the inhibitory effects of silencing $\mathrm{BiP}$ under $\mathrm{H}_{2} \mathrm{O}_{2^{-}}$, CSE-, or IL-1 $\beta$-stimulated conditions were more prominent than those under non-stimulated conditions.

In conclusion, our results show that silencing $\mathrm{BiP}$ attenuated inflammation, ROS generation, and adipogenesis in an in vitro model of GO, suggesting that ER stress is involved in GO pathogenesis. Although further studies are required to establish the relevant downstream pathways involved, our findings provide in vitro background evidence suggesting that ER stress is a viable therapeutic target for managing GO. We cannot conclude that the up-regulation of $\mathrm{BiP}$ and its contribution to GO inflammatory pathogenesis is specific to this disease based on our data. Further molecular and immunologic studies are necessary to determine the disease-specific role of BiP in GO. Moreover, it may be helpful to explore the role of ER stress to evaluate the pathogenesis of other chronic orbital inflammatory diseases, such as IgG4-related orbital disease, the pathogeneses of which are not yet completely understood.

\section{Declaration of interest}

The authors declare that there is no conflict of interest that could be perceived as prejudicing the impartiality of the research reported.

\section{Funding}

This research was supported by the Basic Science Research Program through the National Research Foundation of Korea (NRF) funded by the Ministry of Science and ICT (grant number NRF-2020R1C1C1004081).

\section{Acknowledgement}

The author would like to thank Editage (www.editage.co.kr) for English language editing.

\section{References}

Bahn RS 2010 Graves' ophthalmopathy. New England Journal of Medicine 362 726-738. (https://doi.org/10.1056/NEJMra0905750)

Barrera MJ, Aguilera S, Castro I, Gonzalez S, Carvajal P, Molina C, Hermoso MA \& Gonzalez MJ 2018 Endoplasmic reticulum stress in autoimmune diseases: can altered protein quality control and/or unfolded protein response contribute to autoimmunity? A critical review on Sjogren's syndrome. Autoimmunity Reviews 17 796-808. (https://doi.org/10.1016/j.autrev.2018.02.009)

Bartalena L, Baldeschi L, Dickinson AJ, Eckstein A, Kendall-Taylor P, Marcocci C, Mourits MP, Perros P, Boboridis K, Boschi A, et al. 2008 Consensus statement of the European group on Graves' orbitopathy 
(EUGOGO) on management of Graves' orbitopathy. Thyroid 18 333-346. (https://doi.org/10.1089/thy.2007.0315)

Bertolotti A, Zhang Y, Hendershot LM, Harding HP \& Ron D 2000 Dynamic interaction of BiP and ER stress transducers in the unfolded-protein response. Nature Cell Biology 2 326-332. (https:// doi.org/10.1038/35014014)

Blass S, Union A, Raymackers J, Schumann F, Ungethum U, MullerSteinbach S, De Keyser F, Engel JM \& Burmester GR 2001 The stress protein $\mathrm{BiP}$ is overexpressed and is a major $\mathrm{B}$ and $\mathrm{T}$ cell target in rheumatoid arthritis. Arthritis and Rheumatism 44 761-771. (https:// doi.

org/10.1002/1529-0131(200104)44:4<761::AID-ANR132>3.0.CO;2-S)

Bothun ED, Scheurer RA, Harrison AR \& Lee MS 2009 Update on thyroid eye disease and management. Clinical Ophthalmology 3 543-551. (https://doi.org/10.2147/opth.s5228)

Byun JW, Hwang S, Kang CW, Kim JH, Chae MK, Yoon JS \& Lee EJ 2016 Therapeutic effect of protocatechuic aldehyde in an in vitro model of graves' orbitopathy. Investigative Ophthalmology and Visual Science 57 4055-4062. (https://doi.org/10.1167/iovs.15-19037)

Chong WC, Shastri MD \& Eri R 2017 Endoplasmic reticulum stress and oxidative stress: a vicious nexus implicated in bowel disease pathophysiology. International Journal of Molecular Sciences 18771. (https://doi.org/10.3390/ijms18040771)

Corrigall VM, Bodman-Smith MD, Fife MS, Canas B, Myers LK, Wooley P, Soh C, Staines NA, Pappin DJ, Berlo SE, et al. 2001 The human endoplasmic reticulum molecular chaperone $\mathrm{BiP}$ is an autoantigen for rheumatoid arthritis and prevents the induction of experimental arthritis. Journal of Immunology 166 1492-1498. (https://doi.org/10.4049/jimmunol.166.3.1492)

Dandekar A, Mendez R \& Zhang K 2015 Cross talk between ER stress, oxidative stress, and inflammation in health and disease. Methods in Molecular Biology 1292 205-214. (https://doi.org/10.1007/978-14939-2522-3_15)

Douglas RS, Mester T, Ginter A \& Kim DS 2014 Thyrotropin receptor and CD40 mediate interleukin-8 expression in fibrocytes: implications for thyroid-associated ophthalmopathy (an American Ophthalmological Society thesis). Transactions of the American Ophthalmological Society 112 26-37.

Flamment M, Hajduch E, Ferre P \& Foufelle F 2012 New insights into ER stress-induced insulin resistance. Trends in Endocrinology and Metabolism 23 381-390. (https://doi.org/10.1016/j.tem.2012.06.003)

Garrity JA \& Bahn RS 2006 Pathogenesis of graves ophthalmopathy: implications for prediction, prevention, and treatment. American Journal of Ophthalmology 142 147-153. (https://doi.org/10.1016/j. ajo.2006.02.047)

Harding HP, Novoa I, Zhang Y, Zeng H, Wek R, Schapira M \& Ron D 2000 Regulated translation initiation controls stress-induced gene expression in mammalian cells. Molecular Cell 6 1099-1108. (https:// doi.org/10.1016/s1097-2765(00)00108-8)

Hondur A, Konuk O, Dincel AS, Bilgihan A, Unal M \& Hasanreisoglu B 2008 Oxidative stress and antioxidant activity in orbital fibroadipose tissue in Graves' ophthalmopathy. Current Eye Research 33 421-427. (https://doi.org/10.1080/02713680802123532)

Hotamisligil GS 2010 Endoplasmic reticulum stress and the inflammatory basis of metabolic disease. Cell 140 900-917. (https:// doi.org/10.1016/j.cell.2010.02.034)

Kaufman RJ 1999 Stress signaling from the lumen of the endoplasmic reticulum: coordination of gene transcriptional and translational controls. Genes and Development 13 1211-1233. (https://doi. org/10.1101/gad.13.10.1211)

Khong JJ, McNab AA, Ebeling PR, Craig JE \& Selva D 2016 Pathogenesis of thyroid eye disease: review and update on molecular mechanisms. British Journal of Ophthalmology 100 142-150. (https://doi. org/10.1136/bjophthalmol-2015-307399)

Kim I, Xu W \& Reed JC 2008 Cell death and endoplasmic reticulum stress: disease relevance and therapeutic opportunities. Nature
Reviews: Drug Discovery 7 1013-1030. (https://doi.org/10.1038/ nrd2755)

Kim CY, Lee HJ, Chae MK, Byun JW, Lee EJ \& Yoon JS 2015 Therapeutic effect of resveratrol on oxidative stress in graves' orbitopathy orbital fibroblasts. Investigative Ophthalmology and Visual Science $\mathbf{5 6}$ 6352-6361. (https://doi.org/10.1167/iovs.15-16870)

Kim SE, Lee JH, Chae MK, Lee EJ \& Yoon JS 2016 The role of sphingosine-1-phosphate in adipogenesis of graves' orbitopathy. Investigative Ophthalmology and Visual Science 57 301-311. (https:// doi.org/10.1167/iovs.15-17863)

Ko J, Chae MK, Lee JH, Lee EJ \& Yoon JS 2017 Sphingosine-1-phosphate mediates fibrosis in orbital fibroblasts in graves' orbitopathy. Investigative Ophthalmology and Visual Science 58 2544-2553. (https:// doi.org/10.1167/iovs.16-20684)

Ko J, Kim JY, Lee EJ \& Yoon JS 2018 Inhibitory effect of idelalisib, a selective phosphatidylinositol 3-kinase delta inhibitor, on adipogenesis in an in vitro model of Graves' orbitopathy. Investigative Ophthalmology and Visual Science 59 4477-4485. (https:// doi.org/10.1167/iovs.18-24509)

Ko J, Kim JY, Kim JW \& Yoon JS 2020 Anti-oxidative and antiadipogenic effects of caffeine in an in vitro model of Graves' orbitopathy. Endocrine Journal 67 439-447. (https://doi.org/10.1507/ endocrj.EJ19-0521)

Lee WS, Sung MS, Lee EG, Yoo HG, Cheon YH, Chae HJ \& Yoo WH 2015 A pathogenic role for ER stress-induced autophagy and ER chaperone GRP78/BiP in T lymphocyte systemic lupus erythematosus. Journal of Leukocyte Biology 97 425-433. (https://doi. org/10.1189/jlb.6A0214-097R)

Lee JS, Kim J, Lee EJ \& Yoon JS 2019 Therapeutic effect of curcumin, a plant polyphenol extracted from curcuma longae, in fibroblasts from patients with Graves' orbitopathy. Investigative Ophthalmology and Visual Science 60 4129-4140. (https://doi.org/10.1167/iovs.19-27376)

Lightfoot AP, Nagaraju K, McArdle A \& Cooper RG 2015 Understanding the origin of non-immune cell-mediated weakness in the idiopathic inflammatory myopathies - potential role of ER stress pathways. Current Opinion in Rheumatology 27 580-585. (https://doi. org/10.1097/BOR.0000000000000212)

Maiuri MC, Zalckvar E, Kimchi A \& Kroemer G 2007 Self-eating and selfkilling: crosstalk between autophagy and apoptosis. Nature Reviews: Molecular Cell Biology 8 741-752. (https://doi.org/10.1038/nrm2239)

Malhotra JD \& Kaufman RJ 2007 Endoplasmic reticulum stress and oxidative stress: a vicious cycle or a double-edged sword? Antioxidants and Redox Signaling 9 2277-2293. (https://doi. org/10.1089/ars.2007.1782)

Meng S, Zhang W, Guan LJ, Muhali FS, Zhou JZ, Song RH, Xu J \& Zhang JA 2017 Proteomic analysis reveals aberrant expression of CALR and HSPA5 in thyroid tissues of Graves' disease. Clinical Biochemistry 50 40-45. (https://doi.org/10.1016/j. clinbiochem.2016.08.014)

Park YJ, Yoo SA \& Kim WU 2014 Role of endoplasmic reticulum stress in rheumatoid arthritis pathogenesis. Journal of Korean Medical Science 29 2-11. (https://doi.org/10.3346/jkms.2014.29.1.2)

Prattes S, Horl G, Hammer A, Blaschitz A, Graier WF, Sattler W, Zechner R \& Steyrer E 2000 Intracellular distribution and mobilization of unesterified cholesterol in adipocytes: triglyceride droplets are surrounded by cholesterol-rich ER-like surface layer structures. Journal of Cell Science 113 2977-2989.

Ron D \& Walter P 2007 Signal integration in the endoplasmic reticulum unfolded protein response. Nature Reviews: Molecular Cell Biology $\mathbf{8}$ 519-529. (https://doi.org/10.1038/nrm2199)

Todd DJ, Lee AH \& Glimcher LH 2008 The endoplasmic reticulum stress response in immunity and autoimmunity. Nature Reviews: Immunology 8 663-674. (https://doi.org/10.1038/nri2359)

Tu BP \& Weissman JS 2004 Oxidative protein folding in eukaryotes: mechanisms and consequences. Journal of Cell Biology 164 341-346. (https://doi.org/10.1083/jcb.200311055) 
Ulianich L, Terrazzano G, Annunziatella M, Ruggiero G, Beguinot F \& Di Jeso B 2011 ER stress impairs MHC class I surface expression and increases susceptibility of thyroid cells to NK-mediated cytotoxicity. Biochimica et Biophysica Acta 1812 431-438. (https://doi. org/10.1016/j.bbadis.2010.12.013)

Wakelkamp IM, Bakker O, Baldeschi L, Wiersinga WM \& Prummel MF 2003 TSH-R expression and cytokine profile in orbital tissue of active vs. inactive Graves' ophthalmopathy patients. Clinical Endocrinology 58 280-287. (https://doi.org/10.1046/j.1365-2265.2003.01708.x)

Woodward AM, Di Zazzo A, Bonini S \& Argueso P 2020 Endoplasmic reticulum stress promotes inflammation-mediated proteolytic activity at the ocular surface. Scientific Reports 10 2216. (https://doi. org/10.1038/s41598-020-59237-3)

Yoneda T, Imaizumi K, Oono K, Yui D, Gomi F, Katayama T \& Tohyama M 2001 Activation of caspase-12, an endoplastic reticulum (ER) resident caspase, through tumor necrosis factor receptorassociated factor 2-dependent mechanism in response to the ER stress. Journal of Biological Chemistry 276 13935-13940. (https://doi. org/10.1074/jbc.M010677200)

Yoon JS, Chae MK, Jang SY, Lee SY \& Lee EJ 2012 Antifibrotic effects of quercetin in primary orbital fibroblasts and orbital fat tissue cultures of Graves' orbitopathy. Investigative Ophthalmology and Visual Science 53 5921-5929. (https://doi.org/10.1167/iovs.12-9646)

Yoon JS, Lee HJ, Chae MK, Lee SY \& Lee EJ 2013 Cigarette smoke extract-induced adipogenesis in Graves' orbital fibroblasts is inhibited by quercetin via reduction in oxidative stress. Journal of Endocrinology 216 145-156. (https://doi.org/10.1530/JOE-12-0257)

Zha BS \& Zhou H 2012 ER stress and lipid metabolism in adipocytes. Biochemistry Research International 2012 312943. (https://doi. org/10.1155/2012/312943)

Zhu G, Ye R, Jung DY, Barron E, Friedline RH, Benoit VM, Hinton DR, Kim JK \& Lee AS 2013 GRP78 plays an essential role in adipogenesis and postnatal growth in mice. FASEB Journal 27 955-964. (https:// doi.org/10.1096/fj.12-213330)

Received in final form 21 September 2020

Accepted 20 October 2020

Accepted Manuscript published online 20 October 2020
(C) 2021 Society for Endocrinology Published by Bioscientifica Ltd. Printed in Great Britain 